\title{
Primeros pasos para la obtención de un software arqueoastronómico: \\ Aplicación al sitio arqueológico de El Puente
}

Eduardo Rodas Javier Mejuto

\section{Resumen}

El estudio arqueoastronómico del sitio arqueológico de El Puente comenzó por el interés en ampliar el conocimiento de este sitio, pobremente estudiado dentro de la zona cultural Maya. Sin embargo, al realizar el estudio cobró importancia la posibilidad de implementar un software arqueoastronómico que reprodujera las características del paisaje tanto geográfico como celeste y de esta manera poder extender el estudio a un número mayor de sitios arqueológicos sin incrementar los costes de una larga campaña de campo esperando poder observar fenómenos astronómicos in situ. Por otro lado, este trabajo muestra las conclusiones del comienzo de la aplicación de este método al sitio arqueológico de El Puente, así como las técnicas que son necesarias implementar para la consecución del mismo.

Palabras clave: Arqueoastronomía, SIG, Software, El Puente

\section{Abstract}

Archaeoastronomical research in El Puente archaeologica Isite, located in western Honduras, has been developed by the authors with the purpose of increasing knowledge of this poorly known archaeological site. In the course of this research, it became important the possibility of designing and implementing anarchaeoastronomical software that could reproduce the characteristics of both local geographical landscape and skyscape, so thatworkcould be extended to a larger number of archaeological sites, withou tincreasing th ecosts of longfieldcampaigns in order to waitforastronomicalphenomenae to happen. The present work shows the conclusions obtained in the initial application of this computational approach to research of El Puente archaeological site, as well as themethods and procedures that are needed for the successful implementation of the proposed software. 
Keywords: Archaeoastronomy, GIS, Software, El Puente.

Eduardo Rodas, (eduardo.rodas@unah.edu.hn). Javier Mejuto, (javier.mejuto@ unah.edu.hn), Departamento de Arqueoastronomía y Astronomía Cultural, Facultad de Ciencias Espaciales, Universidad Nacional Autónoma de Honduras. 


\section{INTRODUCCIÓN}

En estudios realizados en la mayoría de las ciudades de la Mesoamérica prehispánica, se han identificado estructuras cuyo diseño arquitectónico se basó en consideraciones astronómicas (Šprajc, 2003). En el caso de Honduras, en el Parque Arqueológico de Copán se han encontrado alineaciones de este tipo, donde se ha visto la relación existente en las orientaciones y posiciones de estelas y templos con el objetivo de señalar fenómenosy eventos del Sol y del planeta Venus sobre el horizonte local con fines rituales y/o míticos, lo que está ampliamente descrito en varias referencias (Pineda de Carias, Véliz\&Agurcia, 2009) (Zablah, 2009). Otro sitio de interés arqueológico en Honduras es el sitio El Puente, parte importante del patrimonio cultural / histórico / turístico de Honduras, tanto por su cercanía y relación con Copán Ruinas como por su importancia específica (Nakamura\& Torres, 1994). Sin embargo, el sitio ha recibido muy poca atención desde la investigación que aporte nueva información relevante para las instituciones del estado encargadas de su cuidado y promoción como destino turístico. Es por eso que un estudio equivalente aportaría nuevos datos a la discusión.

Este estudio es solo una parte del trabajo de investigación realizado en el año 2014 en el sitio arqueológico de El Puente por uno de los autores, el que hasta esa fecha solo había sido estudiado por arqueólogos japoneses que llegaron a Honduras por medio de la Cooperación Japonesa en el país (JICA), pero en el que la UNAH ha empezado a incursionar también a través del Departamento de Arqueoastronomía y Astronomía Cultural (DAQAC) de la Facultad de Ciencias Espaciales (FACES). Otro aspecto relevante es la inclusión de la orografía de forma cuantitativa a través del uso de las Infraestructuras de Datos Espaciales (IDEs), ya que en el estudio del año 2014 solamente se tuvo en cuenta cualitativamente.

Es bien sabida la relevancia que posee el horizonte local en los estudios arqueoastronómicos (Krupp, 1994), este aspecto requiere técnicas informáticas especializadas para adaptar dichas panorámicas a un software de simulación de movimientos de cuerpos celestes, con el objetivo de recrear fenómenos que pudieron haber sido visibles en tiempos antiguos. Todo esto convierte el estudio de estos aspectos topográficos y topoastronómicos en trabajos laboriosos y en muchas ocasiones económicamente costosos, tornándolos en impracticables en algunos casos. Por tanto, sería sumamente útil contar con una herramienta que pudiera recrear estos panoramas - totalmente dependientes del sitio de observación y que permita estudiar varios sitios de forma rápida y eficaz. Por lo tanto, se propone reproducir de una manera fiel el horizonte local utilizando software libre, a partir de datos obtenidos mediante detección remota -que esté fácilmente accesible a 
través del Internet- para el estudio de sitios arqueológicos desde el punto de vista arqueoastronómico y que sea el punto de partida para un software integrador que sea de utilidad para la investigación en el campo.

\section{METODOLOGÍA}

La metodología empleada para la recolección de los datos de este trabajo se basa en la utilizada típicamente en trabajos geodésicos y en la que también se hace uso de la instrumentación que aparece en la Tabla 1.

Tabla 1: Equipo utilizado para la toma de datos de acimut de diferentes estructuras

\begin{tabular}{|c|c|c|c|c|c|}
\hline No. & Descripcion & Marca & Modelo & Unidades de medición & Precision \\
\hline 1 & Teodolito & Sokkia & Leitz DT6 & $\begin{array}{l}\text { Ángulos horizontales y } \\
\text { verticales: Grados, } \\
\text { minutos y segundos de } \\
\text { arco }\end{array}$ & $\begin{array}{l}\quad \pm 10 \\
\text { segundos } \\
\text { de arco }\end{array}$ \\
\hline \multirow[t]{2}{*}{2} & \multirow[t]{2}{*}{ Estación Total } & \multirow[t]{2}{*}{ Nikon } & \multirow[t]{2}{*}{ Top Gun D50 } & $\begin{array}{l}\text { Ángulos horizontales y } \\
\text { verticales: Grados, } \\
\text { minutos y segundos de arco }\end{array}$ & $\begin{array}{c} \pm 10 \\
\text { segundos } \\
\text { de arco }\end{array}$ \\
\hline & & & & Distancia: pies & $\begin{array}{c} \pm 0.005 \\
\text { pies }\end{array}$ \\
\hline
\end{tabular}

En el trabajo de campo se midieron distancias desde el punto de observación hasta puntos específicos de las estructuras estudiadas, con el objetivo de localizarlas espacialmente y así obtener representaciones graficas de dichas estructuras. Asimismo se obtuvieron datos de acimut, el cual expresa numéricamente tanto la orientación de paredes (lienzos), ejes principales de estructuras, edificios 0 monumentos con respecto al punto cardinal Norte, así como las posiciones en el horizonte donde ocurren los ortos u ocasos de los cuerpos celestes. Se ilustran algunos ejemplos de esto utilizando al Sol, cuerpo celeste de mucha importancia para la cultura maya.

Para la etapa de medición del acimut y las distancias de/entre las estructuras del Sitio Arqueológico se planeó la utilización como mínimo, de un teodolito, el cual es un instrumento esencial por su precisión y exactitud a la hora de medir la variable planteada. También se contó con la disponibilidad de una Estación Total, que no es más que un teodolito que tiene la capacidad adicional de medir distancias desde el punto de observación hasta el punto de la estructura cuyas orientaciones se están determinando. Los detalles técnicos de dichos instrumentos se presentan en la Tabla 1. 
Para la calibración de estos equipos, es necesario ubicar primero el Norte de forma precisa y exacta y a partir de allí realizar las mediciones de acimut, para lo que se utilizó el método propuesto en (Mejuto, 2013). Posteriormente, el equipo estuvo listo para la referenciación geográfica de las diversas estructuras del sitio.

Con los datos así obtenidos se evaluó en primer lugar qué alineamientos de lienzos, estructuras o edificios eran más plausibles respecto a fenómenos solares antes de proceder a un análisis detallado de los mismos. Para esto se desarrolló un software especial para crear proyecciones del horizonte local así como de las rutas que siguen diversos astros en la esfera celeste en gráficos circulares (es decir, una proyección en coordenadas polares) que representen fácilmente la orientación de edificios y estructuras respecto a las posiciones de astros en el cielo así como con sus ortos y ocasos en el horizonte, como se detalla en la referencia (Zotti\&Göller, 2005). En dichos gráficos, el observador está ubicado en el centro de un círculo. El perímetro de este círculo representa el horizonte ideal del observador, es decir, un horizonte de altura cero. Otros círculos concéntricos de mayor diámetro representan las alturas sobre el horizonte, los más cercanos al horizonte ideal representan incrementos en altura equivalente a un grado por sobre éste y a partir de los 10 grados de altura, se utilizan círculos que representan incrementos de 10 grados, hasta llegar al más externo que equivale a los 90 grados de altura, es decir, el cenit del observador. En estos círculos concéntricos externos al horizonte ideal se dibuja el horizonte local verdadero, con su altura sobre el horizonte ideal, así como la trayectoria del Sol para efectos del presente estudio, (ver figura 1). 


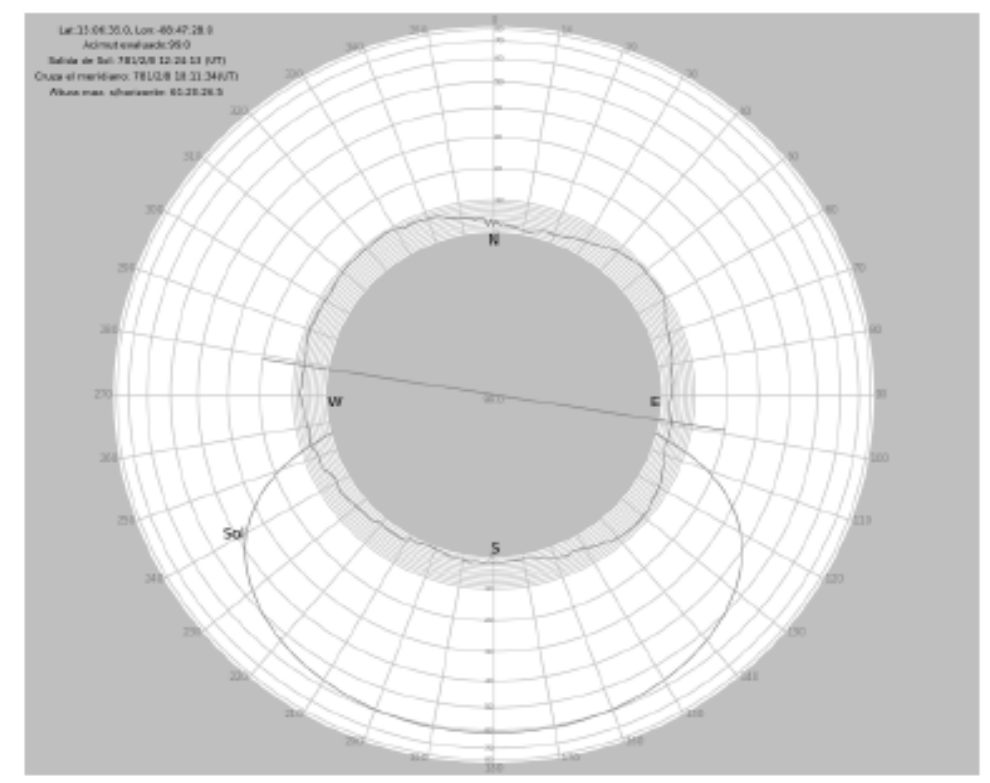

Figura 1: Gráfico circular sobre el que se proyectan orientaciones de lienzos de muros, estructuras y edificios en relación a los movimientos de cuerpos celestes, en este caso, una estructura con orientación hacia los 99 grados de acimut, en relación a la ruta del Sol por la esfera celeste en el 8 de Febrero de 1781 d.C. La figura irregular alrededor del círculo central muestra el horizonte local.

Como en todo estudio que involucra la observación astronómica, es necesario contar con las coordenadas del sitio de observación, así como con la fecha y hora de la observación. Además, el software tiene la capacidad de generar una línea que represente el acimut del componente del resto arqueológico se desea estudiar. En el software desarrollado, se ingresan estos datos en las ventanas correspondientes (ver figura 2). 


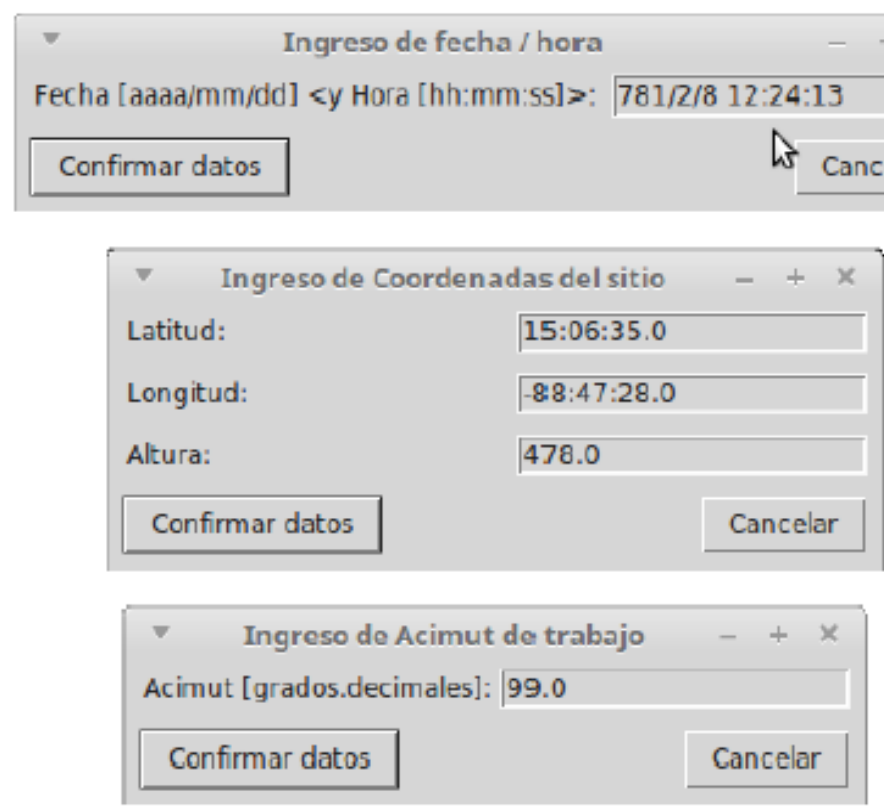

Figura 2: Ventanas que genera el software para el ingreso de los datos requeridos de fecha, hora, lugar y acimut que se desea evaluar en el gráfico circular de análisis de orientaciones

Se considera que existe un probable alineamiento astronómico en el sitio si el orto u ocaso del Sol coincide en un rango de más o menos 2 grados sexagesimales con la línea de orientación de un lienzo o la de la línea que subtienden dos puntos importantes entre dos edificios, proyectado en el horizonte. En los gráficoscirculares que el software elabora, la orientación de un lienzo o estructura bajo estudio se representa como una línea que va desde una altura de 10 grados de un lado del horizonte, hasta que cruza nuevamente el horizonte por el lado opuesto, extendiéndose hasta los 10 grados de altura de dicho lado opuesto. Si proyectamos la ruta que sobre el cielo sigue el Sol, es fácil identificar cualquier punto de intersección entre la línea de orientación bajo estudio y la ruta del cuerpo astronómico de interés, indicando así posibles alineamientos entre la(s) estructura(s) y dicho cuerpo astronómico en puntos cercanos al horizonte.El software que permitió este análisis fue codificado en el lenguaje Python, versión 2.7.6 y se incorporaron rutinas para determinar posiciones del Sol en forma gráfica, gracias al uso de la biblioteca PyEphem (en su versión 3.7.5.3), con la que se tiene la capacidad de calcular las posiciones de cuerpos en el Sistema Solar con la precisión y exactitud requeridas para aplicaciones de grado científico (disponible y explicado en el sitio web http://rhodesmill.org/pyephem). Con el software elaborado se pueden 
identificar visualmente los alineamientos más interesantes desde el punto de vista arqueoastronómico, que tengan las estructuras bajo estudio con los fenómenos y cuerpos celestes y cuyo análisis en detalle se puede realizar posteriormente con datos de efemérides precisas. Es aquí donde cobra importancia la capacidad de reproducir fielmente el horizonte local de lugar, ya que el acimut de un cuerpo astronómico en el horizonte sufre variaciones dependiendo de la altura que éste tenga sobre el horizonte ideal (es decir, un horizonte sin ninguna obstrucción por montañas, árboles o edificios). Esto se puede visualizar en la figura 3.

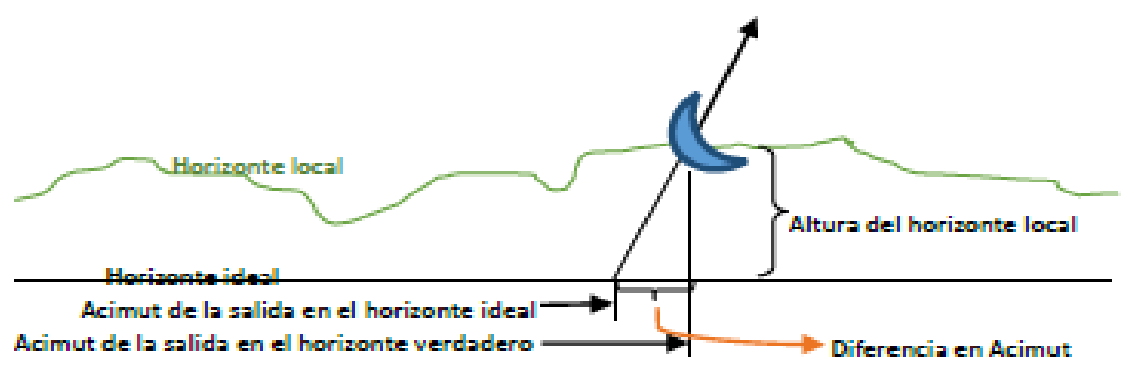

Figura 3: Diferencias en el acimut debido al horizonte local (Diagrama elaborado por los autores)

Para lograr representar el horizonte local del sitio, se puede proceder de dos maneras. La primera es semi-empírica, ya que consiste en tomar una foto panorámica de todo el horizonte, referenciar la misma respecto a los puntos cardinales, utilizar un software de tratamiento de imágenes para remover lo que no se requiere del mismo, tales como el cielo y el suelo. Posteriormente se superpone la imagen sobre la gráfica de salidas y puestas de los astros bajo estudio y se determinan a partir de este montaje los acimutes de los diversos fenómenos astronómicos, para hacer las respectivas comparaciones con los datos de acimut de las estructuras bajo estudio. Una segunda forma de proceder es reproducir el horizonte local por medio de los datos de alturas de los diferentes obstáculos que están en los alrededores del sitio bajo estudio y representarlos en una gráfica, uniendo los diferentes puntos correspondientes a las alturas con una línea. De esta manera, se obtiene una representación cuantitativa y fiel del horizonte tal como se observa desde el sitio. Con este propósito, se obtuvo acceso a la base de datos de alturas que se generó en el proyecto Shuttle Radar TopographyMission (SRTM) de la NASA y con esta información se reconstruyó el horizonte local.A partir del mismo se pueden obtener de forma realista los datos de acimut correspondientes a salidas y puestas del Sol en el sitio arqueológico. 
Para obtener de forma cuantitativa las diferencias entre alineaciones y fenómenos astronómicos sobre el horizonte, se procede, siempre con la ayuda del software desarrollado por los investigadores, a elaborar una tabla de posiciones extremas (es decir, más al Norte o más al Sur de los puntos cardinales Este y Oeste) de la Luna y de Venus, en el período comprendido entre los años 426 d.C. y 900 d.C. (período clásico en Mesoamérica) que es cuando se estima que este sitio arqueológico fue ocupado por los mayas y la cercana ciudad de Copán tuvo influencia sobre este asentamiento.

Posteriormente, se compararon dichos valores con las alineaciones de diversas estructuras, lienzos einter-edificios, con los valores obtenidos en las tablas de posiciones extremas que el Sol alcanza en diferentes fechas del año.

\section{DISCUSIÓN}

Con la metodología descrita anteriormente, se obtuvieron los datos para lienzos, esquinas, portales y escalinatas de las estructuras en mejor estado de conservación en el sitio arqueológico y que están ubicadas en la parte central del mismo. En la figura 4 se muestra una fotografía por satélite del sitio arqueológico, en el que se enmarca en un rectángulo el área estudiada, dentro de la cual se ubicó el teodolito y la estación total. 


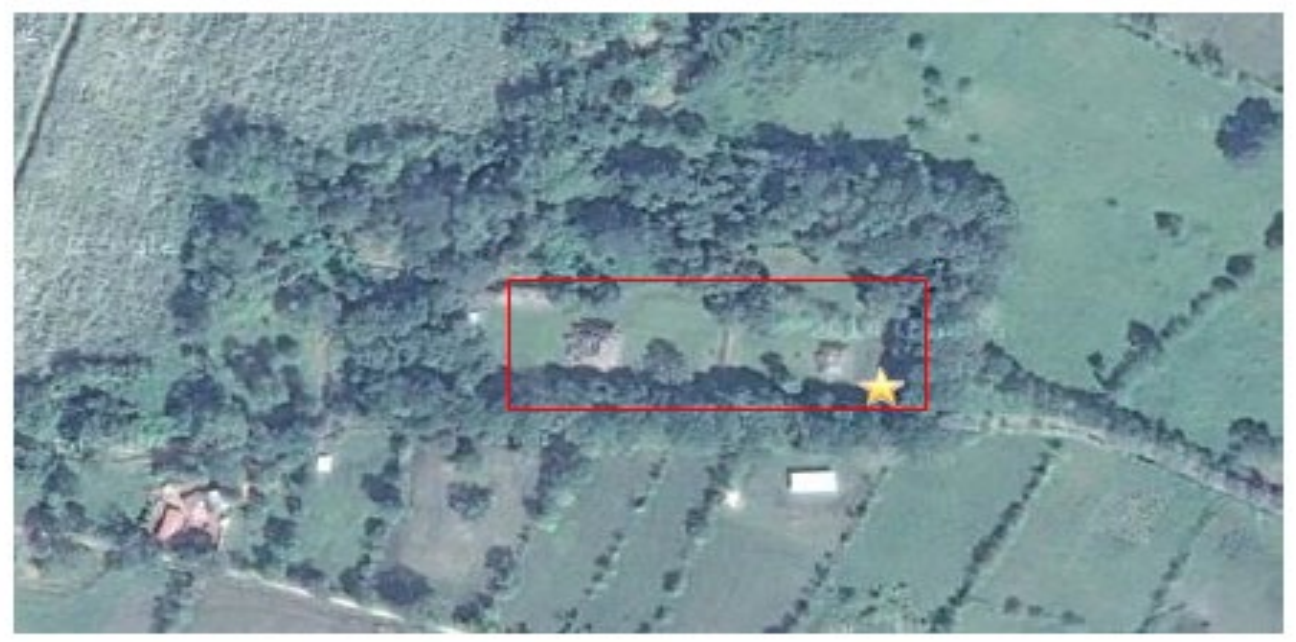

Figura 4: Fotografía satelital del Sitio Arqueológico El Puente, encuadrando el área de estudio (Fuente: Google Maps)

Los datos medidos se presentan de forma gráfica en proyecciones en coordenadas polares (diferentes de las proyecciones generadas con el software previamente descrito) en las figuras 5,7 y 8 , utilizando como referencia para distancia y acimut el punto en donde se ubicó el instrumento de medición. Las distancias están dadas en metros desde el instrumento y el Norte Geográfico marca el Acimut de 0 grados y está localizado en la parte superior de la figura. Los datos numéricos pueden verse en la Tabla 2, en los que se ha tenido en cuenta la codificación mostrada en la figura 6 . Con ello, se pudo establecer la relación entreacimutesy los lados y esquinas de las estructuras. 


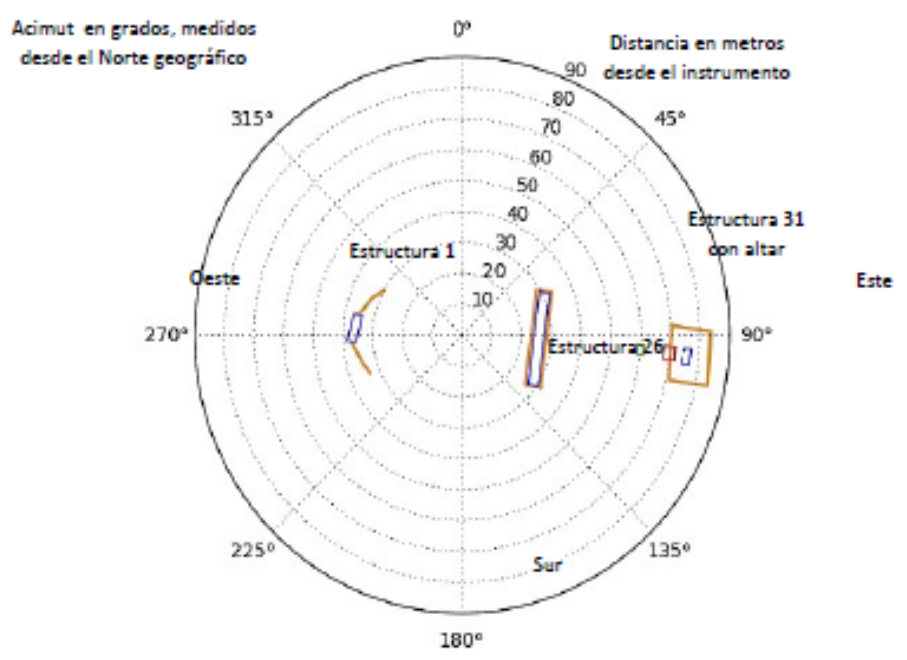

Figura 5: Representación de las estructuras del Sitio Arqueológico El Puente a partir de los datos espaciales obtenidos con el método descrito.

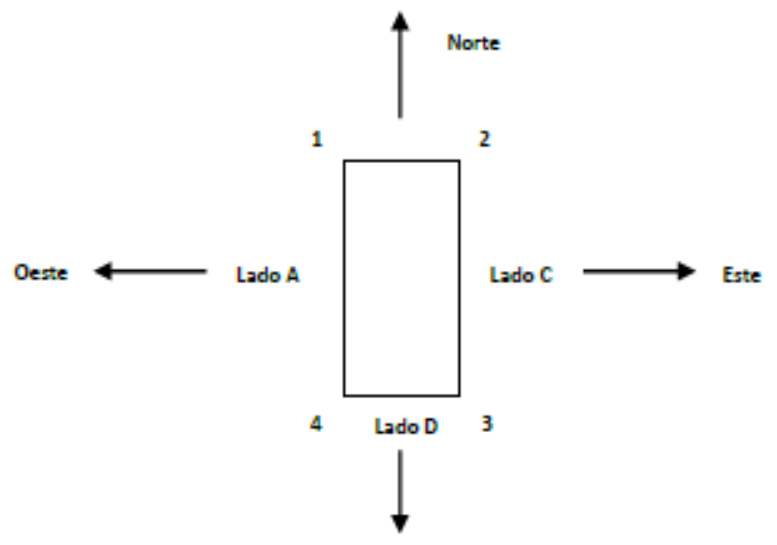

Figura 6: Claves utilizadas para las esquinas medidas y de los lados de los lados de las estructuras así como su posición respecto a los puntos cardinales 
Matemáticamente estas relaciones entre acimutesse expresan mediante la ecuación aplicable a pares de puntos, obtenida a partir de la aplicación de la geometría:

$$
\alpha=\tan ^{-1} \frac{D_{2} \operatorname{sen}\left(z_{2}\right)-D_{1} \operatorname{sen}\left(z_{1}\right)}{D_{2} \cos \left(z_{2}\right)-D_{1} \cos \left(z_{1}\right)} \quad(\text { Ecuación 1) }
$$

Donde:

$a=$ Acimut de la línea que une a los puntos 1 y 2

D1= Distancia del instrumento de medición al punto 1

D2= Distancia del instrumento de medición al punto 2

z1= Acimut del punto 1, en relación al instrumento de medición z2 = Acimut del punto 2, en relación al instrumento de medición 
Tabla 2: Datos obtenidos de las diferentes estructuras estudiadas

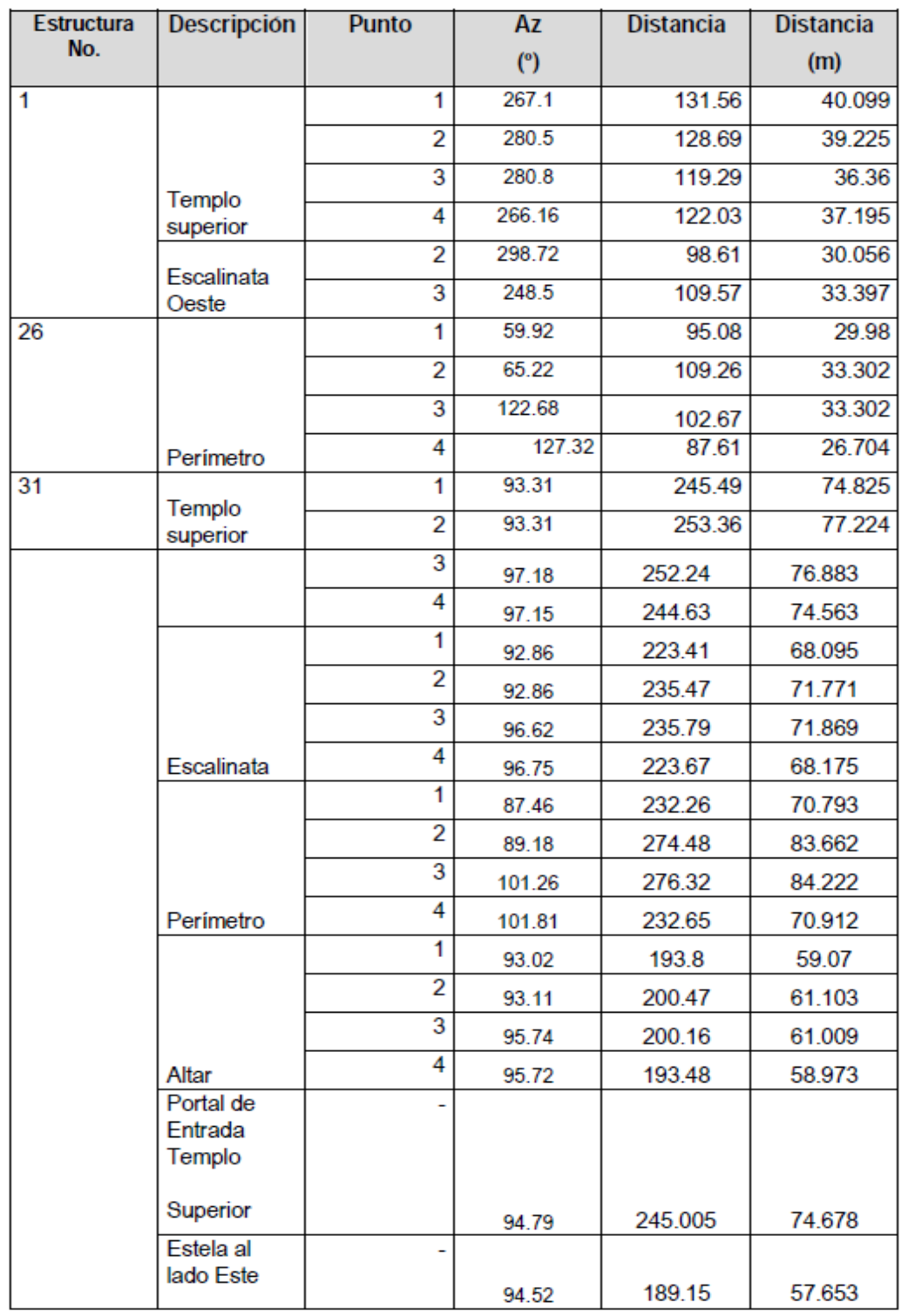


Es importante resaltar que debe corregirse el ángulo a obtenido según los signos del numerador y del denominador de la ecuación 1. Estos datos se presentan en la Tabla 3. A cada alineación se le designó con un número romano, que utilizaremos de ahora en adelante para referirnos a ellas y de las que se muestran algunas en forma gráfica en la figura 6 , mientras que en la figura 6 se muestran los alineamientos entre estructuras.

Se procedió a ingresar la información de cada uno de los acimutes en el software desarrollado para tal efecto. Dado que es sabido que los eventos solares fueron importantes para la cultura maya (Aveni, 2005), se comenzó haciendo hincapié en aquellas partes de estructuras que tuvieran una orientación cercana al orto y ocaso del sol teniendo en cuenta el horizonte local. Para ello se utilizó el año 781 d.C. como referencia temporal ya que éste abarca a la fecha en Cuenta Larga Maya 9.17.10.7.0 (correspondiente a la fecha exacta: 19 de Abril del 781 d.C., utilizando la correlación GMT), inscrita en una estela encontrada cerca del Sitio El Puente por el arqueólogo SylvanusMorley. Se calcularon las diferencias entre los ortos locales del Sol en las fechas de los cinco eventos importantes del Sol (Solsticio de Verano, Solsticio de Invierno, Equinoccio de Primavera, Equinoccio de Otoño y día del paso del Sol por el cenit local), así como las diferencias entre las puestas del Sol y los alineamientos de las estructuras, también para las mismas fechas antes descritas. Los resultados se resumen en la Tabla 4, donde se muestran las diferencias entre el acimut de la alineación bajo estudio respecto al acimut del sol que sale o se pone en la fecha específica, identificando una alineación si esta diferencia es menor a 2 grados. Se provee como referencia el acimut del Sol en cada evento del año, según el horizonte local. Entre paréntesis aparece la altura, en grados sexagesimales, del horizonte local por sobre el horizonte ideal (es decir, sin ningún obstáculo visible en el paisaje) que es de 0 grados. 
Tabla 3: Acimut de cada uno de los lados de las estructuras estudiadas y de otras alineaciones entre estructuras

\begin{tabular}{|c|c|c|c|c|}
\hline Estructura No. & Descripcion & Lado & Acimut $\left({ }^{\circ}\right)$ & Alineacion \\
\hline \multirow[t]{5}{*}{1} & \multirow{3}{*}{$\begin{array}{l}\text { Templo } \\
\text { superior }\end{array}$} & A & $9.16 / 189.16$ & I \\
\hline & & B & $96.70 / 276.70$ & II \\
\hline & & C & $8.53 / 188.53$ & IIII \\
\hline & \multirow{2}{*}{$\begin{array}{l}\text { Escalinata } \\
\text { Oeste }\end{array}$} & $\mathrm{D}$ & $98.93 / 278.93$ & IV \\
\hline & & C & $10.02 / 190.02$ & $\mathrm{~V}$ \\
\hline \multirow[t]{4}{*}{26} & \multirow{4}{*}{ Perimetro } & A & $7.13 / 187.13$ & Vl \\
\hline & & B & $96.30 / 276.30$ & VII \\
\hline & & C & $7.20 / 187.20$ & VIII \\
\hline & & D & $97.87 / 277.87$ & IX \\
\hline \multirow[t]{12}{*}{31} & \multirow{4}{*}{$\begin{array}{l}\text { Templo } \\
\text { superior }\end{array}$} & A & $8.23 / 188.23$ & $\mathrm{x}$ \\
\hline & & B & $93.13 / 273.13$ & $\mathrm{XI}$ \\
\hline & & $\mathrm{C}$ & $19.54 / 199.54$ & XII \\
\hline & & $\mathrm{D}$ & $98.25 / 278.25$ & XIII \\
\hline & \multirow{4}{*}{ Escalinata } & A & $3.83 / 183.83$ & XIV \\
\hline & & B & $94.81 / 274.81$ & $\mathrm{XV}$ \\
\hline & & C & $3.58 / 183.58$ & $\mathrm{XVI}$ \\
\hline & & D & $94.27 / 274.27$ & XVIII \\
\hline & \multirow{4}{*}{ Perimetro } & A & $4.25 / 184.25$ & $\mathrm{XVIII}$ \\
\hline & & B & $98.54 / 278.54$ & $\mathrm{XIX}$ \\
\hline & & $C$ & $3.41 / 183.41$ & $X X$ \\
\hline & & $\mathrm{D}$ & $98.30 / 278.30$ & $\mathrm{XXI}$ \\
\hline & \multirow[t]{4}{*}{ Altar } & A & $6.38 / 186.38$ & XXII \\
\hline & & $B$ & $95.69 / 275.69$ & XXIII \\
\hline & & C & $6.36 / 186.36$ & XXIV \\
\hline & & D & $96.38 / 276.68$ & XXV \\
\hline & $\begin{array}{c}\text { Estela-Portal de } \\
\text { Entrada Templo } \\
\text { Superior }\end{array}$ & - & $95.68 / 275.68$ & XXVI \\
\hline \multirow[t]{3}{*}{ ene-31 } & $\begin{array}{l}\text { Portal Templo } \\
\text { Est.31-Centro } \\
\text { Templo Est.1 }\end{array}$ & - & $94.52 / 274.52$ & XXVII \\
\hline & 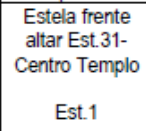 & - & $94.31 / 274.31$ & XXVIIII \\
\hline & $\begin{array}{c}\text { Centro de la } \\
\text { base escalinata } \\
\text { Est.31-Centro } \\
\text { del templo Est.1 }\end{array}$ & - & $94.51 / 274.51$ & XXIX \\
\hline
\end{tabular}




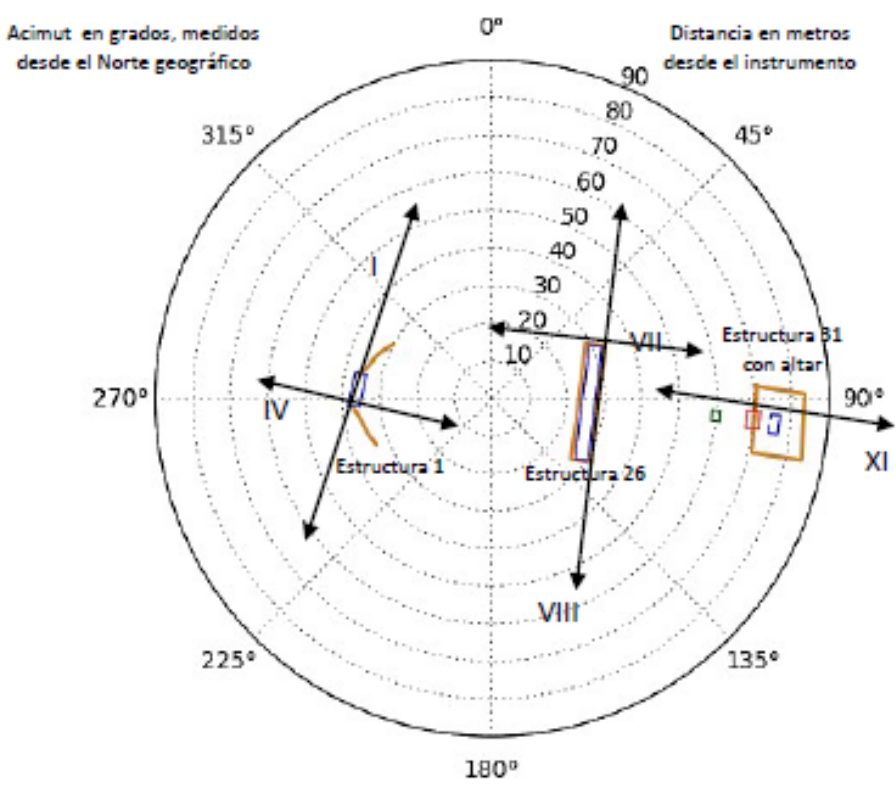

Figura 7: Algunos de los alineamientos de paredes / secciones de estructuras, con las designaciones de cada una de ellas representadas en números romanos, como descrito en la Tabla 3. 


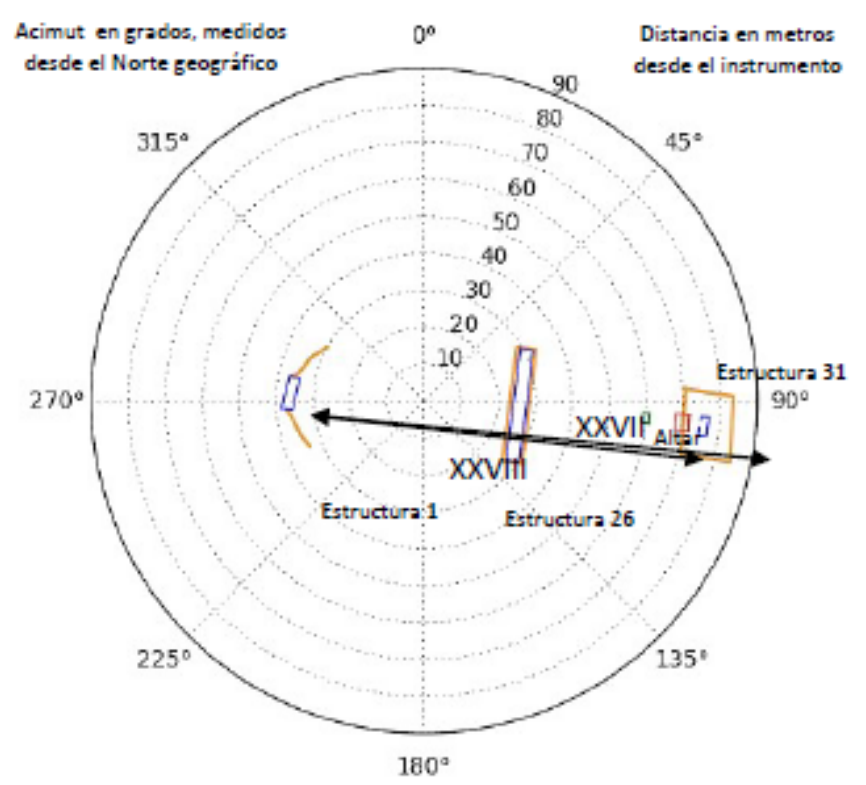

Figura 8: Algunos de los alineamientos entre estructuras, con las designaciones de cada una de ellas representadas en números romanos, como descrito en la Tabla 3. 
Tabla 4. Diferencias de acimut en grados entre las alineaciones y los ortos y ocasos del Sol en las fechas descritas en el sitio arqueológico de El Puente

\begin{tabular}{|c|c|c|c|c|}
\hline $\begin{array}{c}\text { Acimut del Orto } \\
\text { solar }\end{array}$ & $115.26^{\circ}\left(2.5^{\circ}\right)$ & $90.0^{\circ}\left(0^{\circ}\right)$ & $66.36^{\circ}\left(3^{\circ}\right)$ & $74.50^{\circ}\left(2^{\circ}\right)$ \\
\hline $\begin{array}{c}\text { Acimut del Ocaso } \\
\text { solar }\end{array}$ & $244.42^{\circ}\left(3.5^{\circ}\right)$ & $268.65^{\circ}\left(5^{\circ}\right)$ & $\begin{array}{c}292.73^{\circ} \\
\left(6.5^{\circ}\right)\end{array}$ & $284.55^{\circ}\left(6^{\circ}\right)$ \\
\hline $\begin{array}{c}\text { Alineación } \\
\text { (orto/ocaso) } \\
\left({ }^{\circ}\right)\end{array}$ & $\begin{array}{c}\text { Solsticio de } \\
\text { Invierno (WSS) } \\
17 / 12 / 781\end{array}$ & $\begin{array}{c}\text { Equinoccios (EQS) } \\
16 / 3 / 781 \\
18 / 9 / 781\end{array}$ & $\begin{array}{l}\text { Solsticio de } \\
\text { Verano } \\
\text { (SSS) } \\
17 / 6 / 781\end{array}$ & $\begin{array}{c}\text { Pasos del Sol por el } \\
\text { cénit (F/S ZPS) } \\
27 / 4 / 781 \\
8 / 8 / 781\end{array}$ \\
\hline II & $18.56 / 32.28$ & $6.70 / 8.05$ & $30.34 / 16.03$ & $21.81 / 7.37$ \\
\hline IV & $16.33 / 34.51$ & $8.93 / 10.28$ & $32.57 / 13.80$ & $24.04 / 5.10$ \\
\hline VII & $18.96 / 31.88$ & $6.30 / 7.65$ & $29.94 / 16.43$ & $21.41 / 7.77$ \\
\hline IX & $17.39 / 33.45$ & $7.87 / 9.22$ & $31.51 / 14.86$ & $22.98 / 6.20$ \\
\hline $\mathrm{XI}$ & $22.13 / 28.71$ & $3.13 / 4.48$ & $26.77 / 19.60$ & $18.24 / 10.94$ \\
\hline XIII & $17.01 / 33.83$ & $8.25 / 9.60$ & $31.89 / 14.48$ & $23.36 / 5.82$ \\
\hline $\mathrm{XV}$ & $20.45 / 30.39$ & $4.81 / 6.16$ & $28.45 / 17.92$ & $19.92 / 9.26$ \\
\hline XVII & $20.99 / 29.85$ & $4.27 / 5.62$ & $27.91 / 18.46$ & $19.38 / 9.80$ \\
\hline $\mathrm{XIX}$ & $16.72 / 34.12$ & $8.54 / 9.89$ & $32.18 / 14.19$ & $23.65 / 5.53$ \\
\hline $\mathrm{XXI}$ & $16.96 / 33.88$ & $8.30 / 9.65$ & $31.94 / 14.43$ & $23.41 / 5.77$ \\
\hline XXIIII & $19.57 / 31.27$ & $5.69 / 7.04$ & $29.33 / 17.04$ & $20.80 / 8.38$ \\
\hline$X X V$ & $18.88 / 31.96$ & $6.38 / 7.73$ & $30.02 / 16.35$ & $21.49 / 7.69$ \\
\hline $\mathrm{XXVI}$ & $19.58 / 31.26$ & $5.68 / 7.03$ & $29.32 / 17.05$ & $20.79 / 8.39$ \\
\hline XXVII & $20.74 / 30.10$ & $4.52 / 5.87$ & $28.16 / 18.21$ & $19.63 / 9.55$ \\
\hline XXVIII & $20.95 / 29.89$ & $4.31 / 5.66$ & $27.95 / 18.42$ & $19.42 / 9.76$ \\
\hline XXIX & $20.75 / 30.09$ & $4.51 / 5.86$ & $28.15 / 18.22$ & $19.62 / 9.56$ \\
\hline
\end{tabular}

Como puede verse en la tabla 4 las líneas equinocciales y solsticiales distan una gran cantidad de grados de las direcciones que forman las estructuras del sitio arqueológico, se observa que la situación en El Puente es diferente del patrón de orientación de otros sitios arqueológicos ya comentados en la bibliografía. Es probable que esta diferencia se deba a la posible utilización del sitio arqueológico de El Puente,más de carácter comercial, frente a los otros sitios que se centran en un uso principalmente ceremonial. 


\section{Gráficamente esto lo podemos visualizar de la figura 9:}

Diferencia en grados entre acimut de los Solsticios de Invierno (WS - 17/Dic/781 d.C.) y las alineaciones de estructuras en el Sitio Arqueológico El Puente

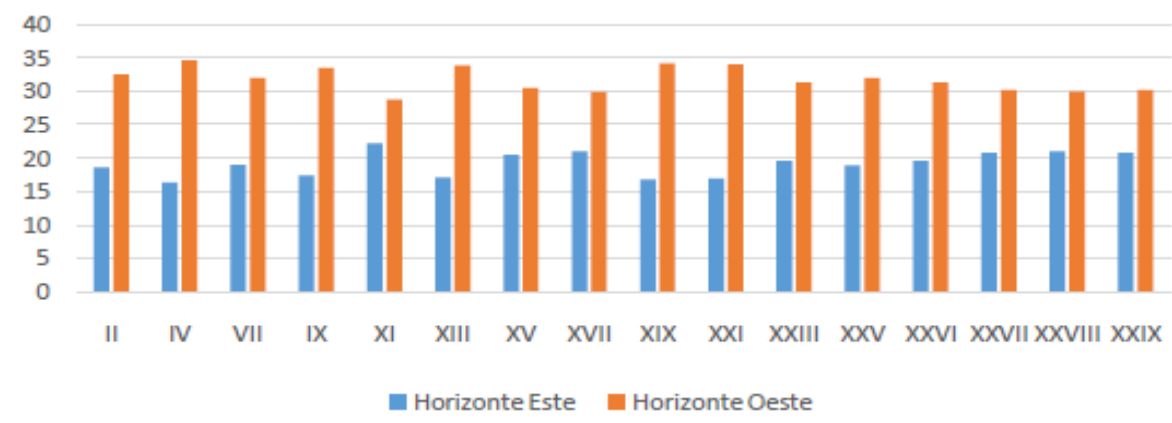

Diferencias entre acimut de los Equinoccios (EQS - 16/Mar/781

d.C. y 19/Sep/781 d.C.) y las alineaciones de estructuras en el Sitio Arqueológico El Puente

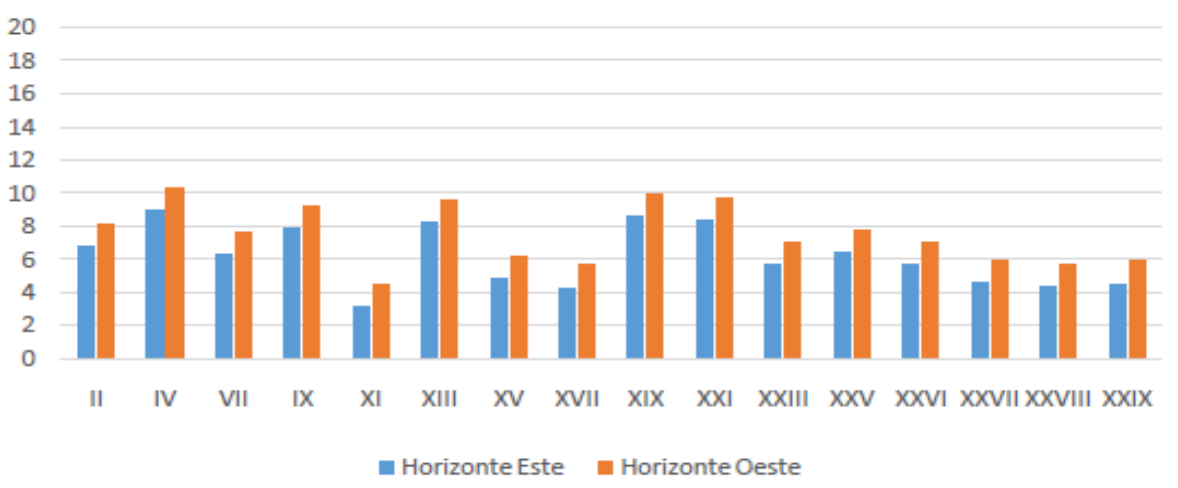



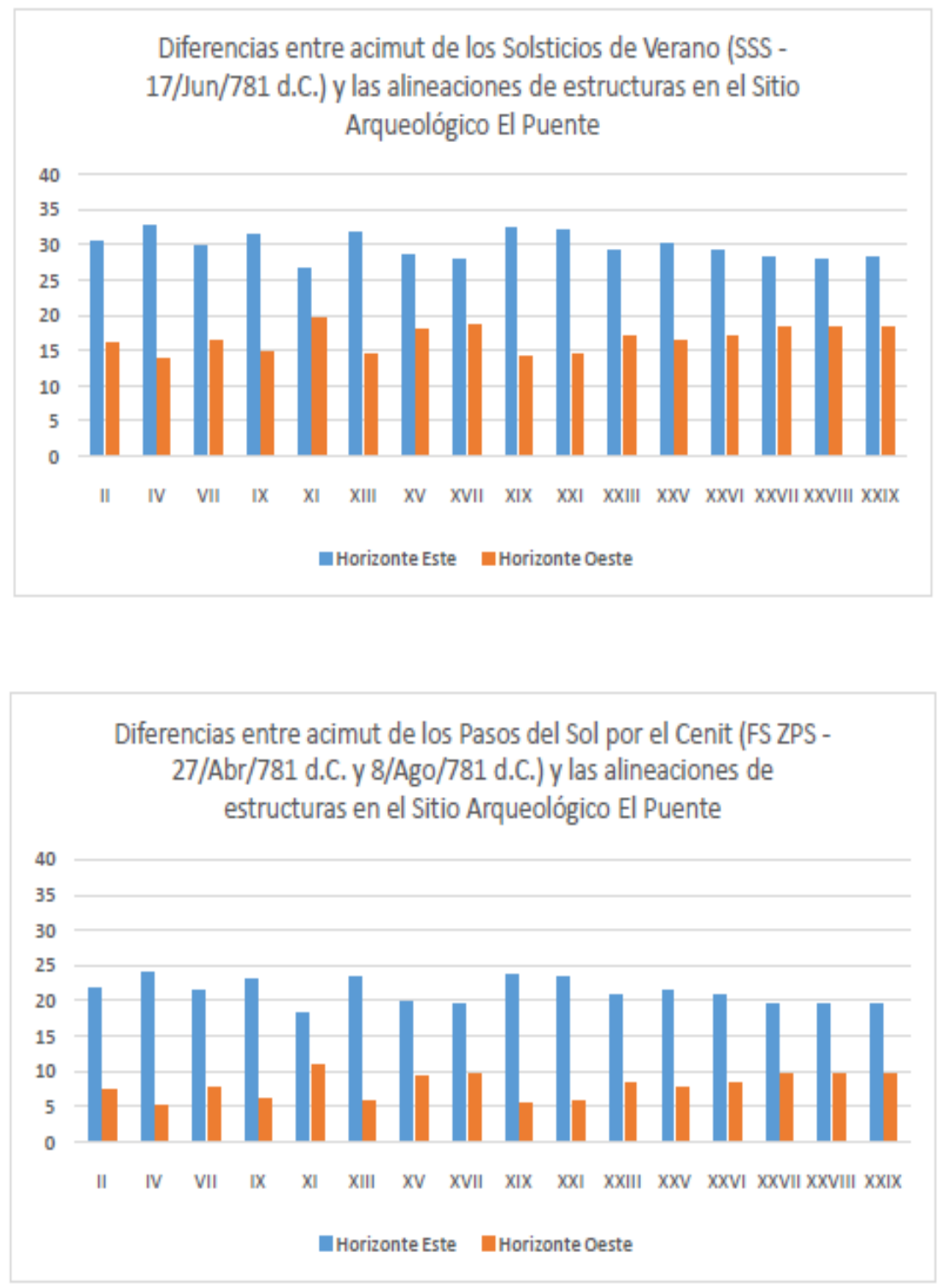

Figura 9: Representaciones gráficas de las diferencias de acimut entre las alineaciones identificadas en el Sitio Arqueológico y los ortos y ocasos del Sol en las fechas descritas 


\section{CONCLUSIÓN}

Desde un punto de vista astronómico no parece haber relación entre las direcciones que forman las estructuras con eventos solares, es por ello que en posteriores estudios se podría ampliar el análisis a eventos lunares y venusianos. Sin embargo, dada la importancia de la ubicación donde se encuentra un sitio arqueológico para el estudio arqueoastronómico se comprobó la necesidad de contar con una herramienta que permita reproducir fielmente el paisaje tanto celeste como geográfico. Ello debe poder realizarse de forma eficiente y eficaz permitiendo a los investigadores el estudio de múltiples casos en busca de patrones culturales.

El paso dado a través de este estudio será el inicial para la consecución de un conjunto de aplicaciones informáticas que faciliten el trabajo en el área de la Arqueoastronomía. Con esta finalidad se realizará un estudio más amplio del sitio y su aplicación a varios sitios arqueológicos del mismo contexto cultural.

\section{BIBLIOGRAFÍA}

- Aveni, A. (2005) La Astronomía y la Arquitectura en la América Antigua y la Cuenca del Mediterráneo (Ed.) Observadores del Cielo en el México Antiguo $(15,311)$ Fondo de Cultura Económica, Universidad Nacional Autónoma de México, Ciudad de México.

- Krupp, E.C. (1994) The Temples WeAlign (Ed.) Echoes of theAncientSkies - TheAstronomy of Lost Civilizations(38, 82, 231, 250-251, 254-255) Dover Publications, Inc.

- Mejuto González, J (2013) Arqueoastronomía: El espacio celeste en la interpretación arqueológica (Tesis de doctorado) Universidad Complutense de Madrid, Madrid, España, (56-59), Disponible en: http://eprints.ucm.es/23111/

- $\quad$ Nakamura, S., Cruz Torres, D. (1993) Investigaciones Arqueológicas y trabajos de restauración en el sitio arqueológico El Puente, Copán, Honduras. V Simposio de Investigaciones Arqueológicas en Copán, (518 - 526) Museo Nacional de Arqueología y Etnología, Guatemala.

- Pineda de Carias, M.C., Véliz, V., AgurciaFasquelle, R. (2009) Estela D: Reloj Solar de la Plaza del Sol del Parque Arqueológico de Copan Ruinas, Honduras, Yaxkin, Año34, XXV (2), pp. 111-136. 
- Šprajc, I. (2003) The South-of-East Skew of Mesoamericanarchitecturalorientations: astronomy and directionalsymbolism. Memorias del Simposio ARQ-13 del 51 Congreso de Americanistas, (161 176). Santiago de Chile.

- Zablah Ávila, J.I. (2009) Búsqueda a través de un Modelo por Ordenador de Alineamientos Astronómicos entre el Planeta Venus, Altares $G$ y Estelas ubicados en la Gran Plaza del Parque Arqueológico de Copán Ruinas, Honduras, (Tesis inédita de Maestría), Universidad Nacional Autónoma de Honduras, Tegucigalpa, Honduras.

- Zotti G., Gröller M.E. (2005) A Sky Dome Visualisationforldentification of AstronomicalOrientations (Ed.) Proceedings IEEE SymposiumonInformationVisualization (9-16) Institute of Electric and ElectronicsEngineers. Disponible en: http://www.cg.tuwien.ac.at/research/ publications/2005/Zotti-2005-vis/ 